Fecha de recepción: diciembre 2018 Fecha de aceptación: febrero 2019 Versión final: mayo 2019

\section{Del diseño industrial al design thinking. Perspectiva histórica de una disciplina en construcción}

Alejo García de la Cárcova *

Resumen: El design thinking - una disciplina relativamente nueva en cuanto a su validación a nivel institucional- surge para suplir las nuevas necesidades del mundo de las corporaciones, por medio de la apropiación de los procesos vinculados al diseño industrial, del cual se desprende.

A partir de una perspectiva histórica de los saberes y metodologías vinculadas a la formación profesional y de enseñanza del diseño industrial, se traza un recorrido que inicia con un amplio conjunto de profesiones que conforman esta nueva disciplina para, a partir del design thinking, volver a expandir los límites del diseño industrial. Como introducción, se revisarán los inicios de la disciplina vinculada a la producción en serie; luego se avanzará en su institucionalización; su actualidad en un mundo globalizado; la conformación y validación del design thinking como heredera del diseño industrial; y por último, su reconocimiento a nivel institucional en el plano de la enseñanza y la práctica profesional en el mundo corporativo.

Palabras clave: Historia - Diseño industrial - Design Thinking - Corporativo - Institucionalización - Cultura - Mercado - Globalización

[Resúmenes en inglés y portugués en las páginas 97-98]

${ }^{(*)}$ Diseñador Industrial. Carrera de Formación Docente (FADU UBA). Docente de la Facultad de Arquitectura, Diseño y Urbanismo, Universidad de Palermo y ORT. Se desempeña en el área de Desarrollo Académico de la Maestría en Gestión del Diseño y Publicaciones Académicas de la Facultad de Diseño y Comunicación de la Universidad de Palermo. Es coordinador de comisiones en el Congreso Latinoamericano de Enseñanza del Diseño (UP).

\title{
Introducción
}

El presente ensayo propone justificar cómo el design thinking -una disciplina relativamente nueva en cuanto a su validación a nivel institucional- surge para suplir las nuevas necesidades del mundo de las corporaciones, por medio de la apropiación de los procesos vinculados al diseño industrial, del cual se desprende. 
Si bien hay autores que trazan los antecedentes del diseño industrial hacia mediados del siglo diecinueve -tal es el caso de Pevsner (2011), Lucie-Smith (1983) y Campi (2007), quienes sugieren su génesis a partir de arquitectos, ingenieros, artistas y artesanos de diferentes gremios, hasta el caso del diseñador y botánico Christopher Dresser-; otros hacen referencia a aquel puñado de personajes de profesión variopinta -ingenieros, arquitectos, decoradores, escenógrafos, e ilustradores, entre otros- surgidos como salvadores de la industria norteamericana posterior a la crisis de 1929-30 (Sparke, 2010). Esto permitirá que destaquen ciertas figuras relacionadas con la conformación de objetos para la vida cotidiana, al punto de que Raymond Loewy -quizás el más ecléctico y prolifero entre todos ellos- llegue a aparecer en la portada de la revista Times en 1949 (Fiell y Fiell, 2003). Pero, tal reconocimiento no será necesariamente para hacer más reconocible a la figura del diseñador industrial y los límites de su función en un mercado de posguerra cada vez más homogéneo y difundido por revistas de interés general, como las especializadas, o también por medio de otros canales de difusión como los museos, o incluso el cine y la televisión. Por otro lado, la enseñanza formal del diseño va a intentar terminar de delinear los imprecisos límites de la disciplina, o al menos brindarle una formación y ciertas metodologías que, con el pasar de los años trascenderán a otros ámbitos del campo empresarial. Para tal caso, instituciones como la alemana Hochschule für Gestaltung de Ulm, la inglesa Royal College of Art, y las norteamericanas Cranbrook Institute y el Institute of Design, serán las primeras en retomar postulados o metodologías heredadas de las escuelas pioneras como la Bauhaus alemana y la Vckutemas rusa (Gay y Samar, 2004). En todas las instituciones mencionadas, serán los arquitectos, con amplia tradición en la formación y profesionalización de su disciplina, quienes llevarán mayormente su dirección.

\section{La validación del diseño}

Para mediados del siglo veinte será principalmente la idea de cultura del diseñador o del diseño -en un amplio y difuso sentido- lo que se asentará en un aspecto culturalmente más reconocible, y siempre mayormente relacionado con cuestiones de carácter fundamentalmente tangibles. Ya sea por medio del diseño industrial y gráfico, como de las más recientes relacionadas con la moda y el interiorismo, el universo objetual será el que se relacione con las disciplinas del diseño.

Habrá que superar los ideales del movimiento moderno, e incluso la breve bisagra de la posmodernidad, para que la globalización de fin de siglo veinte -o Hipermodernismo, como gusta en llamar Lipovetsky (2014)- habilite nuevos roles al diseñador industrial. En tal caso, los consumidores fueron cada vez más conscientes del concepto cultural denominado diseño -no solo a través del consumo de bienes y su función indistintamente de uso o simbólica -en lo que Baudrillard (2014) dio en llamar estructura de colocación-, sino también como parte de una metaestructura que lo envolverá en un todo. Pues el consumo de objetos de uso cotidiano - ¿o debemos decir sencillamente de marcas?- atravesará a todos los estratos sociales: desde las capas más altas, pasando por la inmensa masa de clase media tan propia de los tiempos que corren, hasta los estratos más bajos y carenciados. 
Todos ellos, buscarán suplir el vacío de la sociedad líquida planteada por Bauman (2003), consumiendo productos originales o, en palabras de Morris (2016), sucedáneos, según el poder adquisitivo o el nivel de endeudamiento al que se esté dispuesto a sucumbir, más allá de la clase social a la que se pertenezca. Es así, como lo objetual -física o virtualmente, como se vivencia a partir de la era virtual- se vuelve nocivamente presente. En palabras del filósofo coreano-alemán Han (2015): "La permanente presencia pornográfica de lo visible destruye lo imaginario. Paradójicamente, no da nada a ver." (p.19). Dichos excesos, típicamente de la sociedad capitalista, harán del diseño una necesidad latente y estrechamente vinculada al mundo corporativo.

No obstante y en paralelo a lo antes mencionado, los diseñadores iniciarán un proceso de desmaterialización del producto pero, por sobre todo, de centrar el valor añadido con el que se relaciona por lo general a la disciplina y que se transfiere a los consumidores por medio de los objetos diseñados, y dirigirán su atención directa a las empresas "a las que aportaban nuevos niveles de imaginación, mayor eficacia y mayores beneficios." (Sparke, 2010, p. 198). De esta forma, se fue paulatinamente virando de la cultura del diseño a la cultura de la experiencia, donde los diseñadores destacarán de modo más evidente en la sociedad contemporánea. Si bien en la actualidad conviven ambos conceptos, pues no se puede afirmar que, en tiempos de lo virtual, el mundo objetual y la cultura material han perdido en absoluto su protagonismo. Menos aún, si se piensa en una sociedad vinculada con el individualismo y el egocentrismo, donde destacan figuras del star system. Tal el caso del ya no tan actual Philippe Starck -heredero del podio largamente atribuido a su compatriota Loewy-, como de un puñado de diseñadores internacionales más actuales, generalmente hombres y occidentales.

\section{El diseño en la globalización}

Centrando ahora la atención en la actualidad, sin perder de vista el complejo entramado recién desarrollado, se verá cómo el diseño se encuentra más que nunca en boca de una más amplia mayoría de público que, no solo continua consumiendo productos diseñados, sino también experiencias que se vinculan con la metodología del diseño.

Miren Etxezarreta es explícita al referirse a la disciplina del diseño industrial en el contexto actual capitalista: "el diseño se utiliza principalmente para intentar aumentar la cuota de mercado" (como se cita en Pelta, 2007). Si bien, en las primeras décadas del siglo veintiuno se han reforzado las tendencias ecologistas surgidas a partir de los años sesenta del siglo anterior, lo cierto es que el capitalismo no da tregua ni a sus niveles de producción ni al permanente deterioro del medio ambiente. Sin embargo, esto se da a conocer por el mismo mercado a un público más general que ha comenzado a tomar tímidas cartas en el asunto: siguiendo las tendencias promovidas por el primero, o por acciones de menor escala llevadas a cabo por los segundos.

De cualquier modo, es el mercado y sus corporaciones quienes marcan el norte a seguir, por medio de los canales de comunicación vigentes en la actualidad: tanto tradicionales (televisión y medios gráficos impresos), como los nativos de la era virtual (internet y redes sociales, en dispositivos fijos y móviles). 
Es así como las empresas internacionales buscarán nuevos medios para persuadir a sus consumidores. Y, una vez más, como en la crisis económica norteamericana y de impacto mundial antes mencionada, será el diseño industrial el medio para propiciar este impulso. Como señala Heskett (2002), los diseñadores industriales están accediendo a funciones ejecutivas en cargos relacionados a la estrategia de la organización, no formando solo parte de la concepción de objetos sino también en un plano más amplio del negocio (p.193). Para ello, será crucial la aplicación de la innovación en variados aspectos de la organización. Como mencionan Hill y Jones "En realidad, la innovación con frecuencia es el principal factor en la evolución de la industria y ocasiona el movimiento a través del ciclo de vida de ésta." (2009, p. 64).

Si bien el término innovación se aplica hoy en día a un variado conjunto de situaciones, en el estricto sentido económico se refiere a la exitosa aplicación de una idea en el contexto de una organización "para crear nuevos productos o procesos" (Hill y Jones, 2009). Esto quiere decir que, no basta con la idea per se para que ésta sea innovadora sino que debe poder llevarse a la práctica o materializarse, según el caso. Así, muchas empresas cosecharán sus éxitos por medio de la innovación tecnológica, al presentar nuevos materiales, $\mathrm{u}$ ofrecer un nuevo servicio a sus clientes. Los casos de Uber o Airbnb, que ofrecen nuevas maneras de trasladarse o alojarse, son claros ejemplos de innovación a la hora de viajar. Pero éstos no serían posibles sin las nuevas tecnologías de la era virtual. La posibilidad de resolver éste u otro tipo de situaciones, contando solo con un dispositivo móvil, una aplicación y una conexión a internet, era impensado hace pocas décadas atrás. Estos tres elementos recién mencionados representarán, en gran parte el canal de comunicación y comercialización que irá modificando la experiencia del usuario, en relación al ya tradicional canal de venta o retail.

\section{Design thinking: heredero del diseño industrial}

Pero, volviendo al plano de la innovación, y cómo el diseño industrial es responsable en gran medida de que ésta esté en boca de una gran mayoría de público, se deben tener en cuenta las palabras de uno de los responsables de promover mundialmente el término que da título al presente escrito: design thinking.

Tim Brown, actual CEO y presidente de IDEO -empresa global fundada por David Kelley en 1987 y dedicada a "crear impacto positivo por medio del diseño" (IDEO, 2018)-, formula la siguiente afirmación, a saber:

Lo que necesitamos es un enfoque en innovación que sea poderoso, efectivo y ampliamente accesible, que pueda ser integrado en todos los aspectos del negocio y la sociedad, y que los individuos y los equipos puedan utilizar para generar ideas innovadoras que sean implementadas y que, por consiguiente, tengan impacto. El design thinking (...) ofrece justo ese enfoque. (Brown, 2009, p. 3). 
De tal modo, por medio del design thinking, sentará las bases de una metodología que desarrollará en su compañía, de la mano de su fundador, y que partirá del proceso vinculado a la concepción de nuevos productos o rediseños que forman parte del diseño industrial para ofrecer soluciones innovadoras, como así también en la resolución de problemas más amplios vinculados a los procesos y las experiencias de usuarios, ya sea de consumidores como puertas adentro de una institución o corporación.

Si bien hay muchos autores que tratan sobre el proceso de diseño, como Llovet (1979), Löbach (1981), Lawson (2000), Bürdek (2007), Munari (2008) y Aguirre (2013); o como mejor prefiere definirlo Ricardo Blanco (2007) en términos de estrategia -y para lo cual cobra mayor sentido en el presente escrito- se opta, por definirlo en unos pocos pasos, a saber: Problema, Análisis, Requisitos, Programa, Propuestas, Proyecto, Prototipo y Producto. Cabe destacar que, en las últimas cuatro etapas, se debería o podría verificar e iterar, para lograr un resultado más satisfactorio, real y viable comercialmente.

Partiendo de lo antedicho, los miembros de IDEO proponen ciertos pasos derivados del proceso de diseño pero con un énfasis centrado en el usuario, e incluyendo a un conjunto multidisciplinario de participantes, para resolver un problema propuesto por un determinado cliente. Esto no quiere decir que el diseño industrial no base todo su proceso en una determinada necesidad de un usuario específico. Al referirse históricamente a una metodología conducente a la concreción de un objeto material, este proceso tiende a definir de manera más contundente aquellos elementos relacionados al producto y su fabricación. Esto se refiere puntualmente a lo relacionado con sus materiales y partes constitutivas, como con los procesos de fabricación que formarán parte de su concepción. Para ello, será inevitable acudir a la ayuda de otras y variadas disciplinas que aporten sus conocimientos específicos, para lograr el mejor resultado.

Por el contrario, y siendo el design thinking un proceso que no necesariamente concluirá en un producto $-\mathrm{y}$ mucho menos será llevado a cabo por diseñadores industriales en particular-, sino en un servicio o una experiencia de usuario, tanto real como virtual, es que sus pasos se relacionan con una mirada más blanda, en relación al usuario final. Así, los pasos propuestos para el design thinking, a grandes rasgos y en orden son: Empatizar, Definir, Idear, Prototipar, y Evaluar.

Siendo que estos pasos serán llevados a cabo por personal de las áreas más variadas dentro de una organización - pues de esa diversidad de formaciones y roles se nutre éste procesose sobrentiende que, a tal efecto, se tratará de manera más blanda. Como lo expresa Liedtka (2018), involucrando diversas voces en el proceso conlleva a soluciones superadoras. Y, las variaciones que los diversos actores puedan aportar desde sus saberes específicos, abrirán paso al éxito buscado.

De dicha manera, se podrá replicar, éste u otra variante surgida de otras organizaciones, para aproximarse a resultados más innovadores. Tal es el caso, entre otros, del propuesto por la compañía IBM, y su modelo Enterprise Design Thinking, que propone un "marco de trabajo o framework para entregar grandes experiencias para nuestros usuarios” (IBM, 2018). 


\section{La institucionalización del design thinking}

Actualmente, las instituciones de enseñanza del diseño, como así también de administración de empresas o ciencias económicas, e incluso de otras áreas impensadas pero preocupadas por ofrecer soluciones innovadoras a nivel global, desarrollan programas donde capacitarse en design thinking. A su vez, muchas organizaciones toman dichas capacitaciones para luego trasladar la experiencia puertas adentro.

En algunos casos, como por ejemplo las empresas IBM y Accenture, tiene un área interna que desarrolla este proceso, tanto para sus empleados, como así también para sus clientes. En ambos casos, siempre en función a los proyectos en curso y con intenciones de lograr los resultados más innovadores.

Lo cierto es que, por medio de dicha institucionalización del design thinking, como por vías de su promoción a través de los medios de comunicación especializados y no especializados, se ha difundido este término a un amplio público, lo cual le ha otorgado visibilidad y reconocimiento como nueva disciplina.

\section{Conclusiones}

Podríamos concluir diciendo que el diseño industrial surge de variadas disciplinas, para derivar en una nueva, con sus propias metodologías y procesos; mientras que el design thinkink se desprende del diseño industrial para incorporar y hacer partícipe a las diversas áreas y actores involucrados desconocedores a priori de los procesos de diseño.

Incluso, si bien el design thinking se desprende del diseño industrial, por vías de su proceso de diseño a partir de las búsquedas del equipo de IDEO por satisfacer las demandas del actual mercado globalizado, no todos los responsables en promover y transmitir ésta estrategia de negocio son diseñadores industriales. De hecho, mayormente no lo son, y es esto mismo lo que habilita que el design thinking se constituya como una nueva disciplina o metodología. Más aún, si se tiene en cuenta que, dentro de los pasos involucrados, se pone en juego la creatividad y el uso informal de recursos que se encuentran a mitad de camino entre el arte y el diseño; otro largo debate que aún sigue en discusión. Así es como, dibujo de rápidos bocetos, la creación de maquetas con materiales variados -del tipo empleados en los niveles iniciales de la educación formal- como el recurso de utilizar pos-it, son elementos obligados a la hora de formar parte de este proceso. Otros recursos provenientes de otras disciplinas, incluyen el brainstorming, o incluso la actuación. Todo dicho conjunto de elementos, hace más atractivo el alcance de esta estrategia a un variado público y lo desprenden del diseño industrial.

Otra importante diferencia es que, no solo se emplea en organizaciones e instituciones educativas, sino también lo llevan a cabo organismos gubernamentales, escuelas de nivel medio, como escuelas de arte, ciencias sociales y humanísticas. En definitiva, es un proceso que surge de una especificidad vinculada al diseño objetual y que, hoy día, puede brindar tanto productos como servicios tangible e intangibles, a un amplio espectro de necesidades. De esta manera, el design thinking, si bien surge de una disciplina más de- 
finida y específica, amplía sus fronteras y la vuelve permisible a una variedad impensada de profesiones y oficios.

Así pues, el design thinking y su aplicación en el mundo corporativo y más allá, su conocimiento y validación como disciplina independiente y necesaria para el actual estado de situación de globalización, cobra autonomía y, a su vez, es heredera de las teorías de la arquitectura y el diseño industrial.

Tal como se explicitó, ambas disciplinas tienen un proceso que, aunque puedan describirse con diferentes nombres en cada uno de sus pasos, el desprendimiento desde el diseño industrial se hace más evidente al momento de responder a la necesidad de resolver un problema específico para un usuario determinado.

También, así como se demostró que el diseño industrial surge de un variado conjunto de disciplinas -aunque su mayor influencia parte de la arquitectura-, en el caso del design thinking se constata su nacimiento por vías de los diseñadores industriales y su necesidad de adaptarse a las nuevas demandas del mercado.

A su vez, ambas disciplinas surgen de la máxima aprender haciendo -learning by doing- lo que le dará al design thinking una rápida aceptación en el campo de la educación, gracias a los acelerados medios de difusión propios de la globalización, a las demandas del mercado, y a las instituciones educativas en busca de innovadores y atractivos programas de estudio, que requieren de una participación más activa por parte de sus estudiantes.

Por último, y no menos importante, cabe recordar y resaltar que, tanto el diseño industrial como el design thinking, centran su atención en la tríada persona/usuario/consumidor y su vínculo con lo objetual o su experiencia de usuario. A su vez, los conceptos de estética y belleza, serán de sumo valor para persuadir al momento de adquirir un producto o servicio diseñado para su consumo masivo. Ambos conceptos: vinculo y belleza, podrían resumirse nuevamente en palabras de Han: "La salvación de lo bello es la salvación de lo vinculante". (2015, p.110).

Aspiremos a que, tanto el design thinking, como el diseño industrial, favorezcan un vínculo más sano y humano a esta sociedad.

\section{Listado de Referencias Bibliográficas}

Aguirre, J. M. (2013). Diseño: enseñanza, aprendizaje y ego-axialidad. Córodn: Editorial de la Facultad de Arquitectura, Urbanismo y Diseño de la Universidad Nacional de Córoba. Baudrillard, J. (2014). El sistema de los objetos. México d.f.: grupo editorial siglo veitiuno.

Blanco, R. (2007). Notas sobre diseño industrial. Buenos Aires: Nobuko.

Brown, T. (2009). Change by design: how design thinking transforms organizations and inspires innovation. New York: Harper.

Bürdek, B. E. (2007). Diseño: Historia, teoría y práctica del diseño industrial. Barcelona: Editorial Gustavo Gili.

Campi, I. (2007). La idea y la materia. Vol. 1: El diseño de producto en sus orígenes. Barcelona: Editorial Gustavo Gili.

Fiell Ch. y Fiell, P. (2003). El diseño industrial de la A a la Z. Köln: Taschen.

Gay, A y Samar, L. (2007). El diseño industrial en la historia. Córdoba: ediciones tec. 
Han, B. (2015). La salvación de lo bello. Buenos Aires: Herder.

Heskett, J. (2002). Toothpicks \& Logos. Design in everyday life. Cornwall: Oxford University press.

Hill, C. W. L. \& Jones, G. R. (2009). Administración Estratégica. México D.F.: Mc Graw-Hill.

Lawson, B. (2000). How designers think. The design process demystified. Kent: Architectural press.

Liedtka, J. (2018). Why Design Thinking Works. Disponible en: https.//hbr.org/2018/09/ why-design-thinking-works?referral=03759\&cm_vc=rr_item_page.bottom

Llovet, J. (1997). Ideología y metodología del diseño. Barcelona: Editorial Gustavo Gili.

Löbach, B. (1981). Diseño industrial. Bases para la configuración de los productos industriales. Barcelona: Editorial Gustavo Gili.

Lucie-Smith, E. (1983). A history of industrial design. Golborne: Phaidon.

Munari, B. (1983). ¿Cómo nacen los objetos? Barcelona: Editorial Gustavo Gili.

Pelta, R. (2007). Diseñar con la gente. En G. S. Sol (2012). Diseño, arte, cultura y tecnología. México D.F.: Universidad Autónoma Metropolitana.

Pevsner, N. (2011). Pioneros del diseño moderno: de William Morris a Walter Gropius. Buenos Aires: Infinito.

Sparke, P. (2010). Diseño y cultura: una introducción. Desde 1900 hasta la actualidad. Barcelona: Editorial Gustavo Gili.

\section{Bibliografía}

Aguirre, J. M. (2013). Diseño: enseñanza, aprendizaje y ego-axialidad. Córodn: Editorial de la Facultad de Arquitectura, Urbanismo y Diseño de la Universidad Nacional de Córoba. Baudrillard, J. (2014). El sistema de los objetos. México d.f.: grupo editorial siglo veitiuno. Bauman, Z. (2003). Modernidad líquida. Buenos Aires: Fondo de Cultura Económica.

Blanco, R. (2007). Notas sobre diseño industrial. Buenos Aires: Nobuko.

Brown, T. (2009). Change by design: how design thinking transforms organizations and inspires innovation. New York: Harper.

Bürdek, B. E. (2007). Diseño: Historia, teoría y práctica del diseño industrial. Barcelona: Editorial Gustavo Gili.

Campi, I. (2007). La idea y la materia. Vol. 1: El diseño de producto en sus orígenes. Barcelona: Editorial Gustavo Gili.

Fiell Ch. y Fiell, P. (2003). El diseño industrial de la A a la Z. Köln: Taschen.

Han, B. (2015). La salvación de lo bello. Buenos Aires: Herder.

Gay, A y Samar, L. (2007). El diseño industrial en la historia. Córdoba: ediciones tec.

Heskett, J. (2002). Toothpicks \& Logos. Design in everyday life. Cornwall: Oxford University press.

Hill, C. W. L. \& Jones, G. R. (2009). Administración Estratégica. México D.F.: Mc Graw-Hill.

Lawson, B. (2000). How designers think. The design process demystified. Kent: Architectural press. 
Liedtka, J. (2018). Why Design Thinking Works. Disponible en: https.//hbr.org/2018/09/ why-design-thinking-works?referral $=03759 \& \mathrm{~cm} \_v c=r r \_i t e m \_p a g e . b o t t o m$

Lipovetsky, G. (2014). Los tiempos hipermodernos. Barcelona: Anagrama.

Llovet, J. (1997). Ideología y metodología del diseño. Barcelona: Editorial Gustavo Gili.

Löbach, B. (1981). Diseño industrial. Bases para la configuración de los productos industriales. Barcelona: Editorial Gustavo Gili.

Lucie-Smith, E. (1983). A history of industrial design. Golborne: Phaidon.

Morris, W. (2016). La era del sucedáneo y otros textos contra la civilización moderna. La Rioja, España: Pepitas de calabaza.

Munari, B. (1983). ¿Cómo nacen los objetos? Barcelona: Editorial Gustavo Gili.

Pelta, R. (2007). Diseñar con la gente. En G. S. Sol (2012). Diseño, arte, cultura y tecnología. México D.F.: Universidad Autónoma Metropolitana.

Pevsner, N. (2011). Pioneros del diseño moderno: de William Morris a Walter Gropius. Buenos Aires: Infinito.

Sparke, P. (2010). Diseño y cultura: una introducción. Desde 1900 hasta la actualidad. Barcelona: Editorial Gustavo Gili.

\begin{abstract}
Design thinking - a relatively new discipline in terms of its validation at the institutional level - arises to meet the new needs of the corporate world, through the appropriation of the processes linked to industrial design, from which it emerges.

From a historical perspective of the knowledge and methodologies linked to professional training and teaching of industrial design, a journey is drawn up that begins with a broad set of professions that make up this new discipline to, starting with design thinking, return to expand the limits of industrial design. As an introduction, the beginnings of the discipline linked to serial production will be reviewed; then it will advance in its institutionalization; its present in a globalized world; the conformation and validation of design thinking as heir to industrial design; and finally, its recognition at the institutional level in terms of teaching and professional practice in the corporate world.
\end{abstract}

Keywords: History - Industrial Design - Design Thinking - Corporate - Institutionalization - Culture - Market - Globalization.

Resumo: Design thinking - uma disciplina relativamente nova em termos de sua validação em nível institucional - surge para atender às novas necessidades do mundo corporativo, através da apropriação dos processos ligados ao design industrial, dos quais emerge. A partir de uma perspectiva histórica do conhecimento e metodologias ligadas à formação profissional e ao ensino do design industrial, é elaborada uma jornada que começa com um amplo conjunto de profissões que compõem essa nova disciplina, começando com o design thinking, retornando a expandir os limites do design industrial. Como introdução, o início da disciplina vinculada à produção em série será revisado; então avançará em sua 
institucionalização; seu presente em um mundo globalizado; a conformação e validação do design thinking como herdeiro do design industrial; e, finalmente, seu reconhecimento no nível institucional em termos de ensino e prática profissional no mundo corporativo.

Palavras-chave: História - Desenho Industrial - Design Thinking - Corporativo - Institucionalização - Cultura - Mercado - Globalização.

[Las traducciones de los abstracts fueron supervisadas por el autor de cada artículo] 